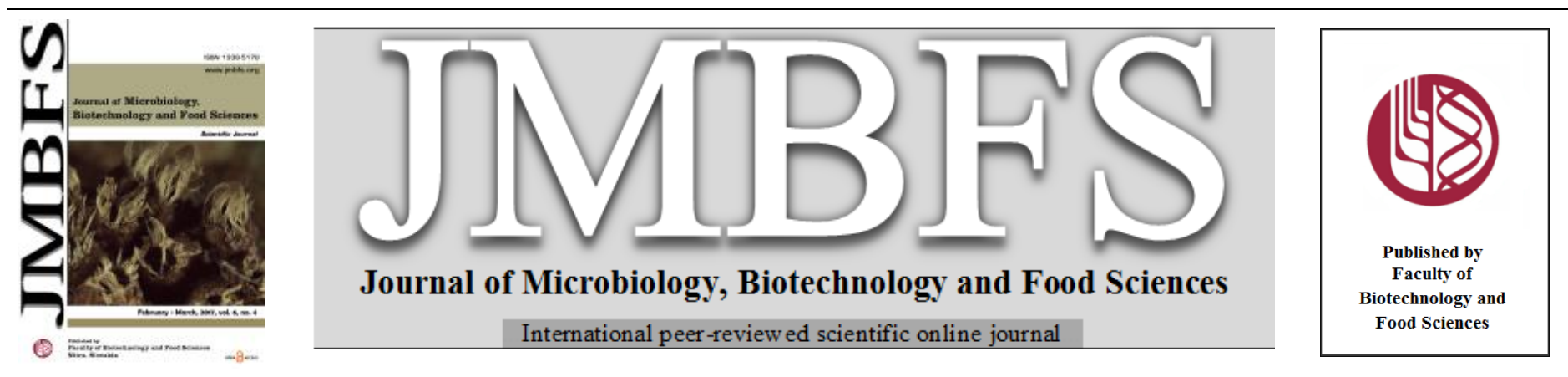

\title{
EFFECTS OF DIFFERENT METHODS OF DRYING ON ANTIOXIDANT AND MICROSCOPIC CHARACTERISTICS OF Spirullina platensis ENRICHED SOY YOGURT
}

\author{
Samadrita Sengupta ${ }^{1}$ and Jayati Bhowal $*^{2}$ \\ Address(es): \\ ${ }^{1}$ IIEST, Shibpur, Research Fellow, SOCSAT, Botanic Garden, 711103 and Howrah, West Bengal, (+91) 9903780850. \\ ${ }^{2}$ IIEST, Shibpur, Assistant Professor, SOCSAT, Botanic Garden, 711103 and Howrah, West Bengal, (+91) 9831672455.
}

*Corresponding author: jayatibhowal@gmail.com

doi: $10.15414 / j m b f s .2017 .6 .4 .1001-1006$

\section{ARTICLE INFO}

Received 13. 10. 2015

Revised 12. 8. 2016

Accepted 13.11. 2016

Published 1. 2. 2017

Regular article

open $\bigodot_{\text {access }}$

\begin{abstract}
Spirulina platensis was discussed as an active compound with regard to the combined effects with soy yogurt in dried food formulation. Drying influenced the microscopic, and antioxidant properties of soy yogurts, and could be used to create new functionalities. The present investigation aimed to convert Spirulina platensis enriched soy yogurts to powder form by different drying methodologies such as air, vacuum, freeze, and microwave drying and evaluate the microstructure and antioxidant activity of the powders obtained. Antioxidant activities were assayed by using polyphenol assay, estimation of carotenoid content, DPPH radical scavenging activity and FRAP assay. Structural changes were analyzed using a scanning electron microscope and an X-ray diffraction pattern. Freeze-drying was produced significantly high quality dried Spirulina platensis enriched soy yogurt, i.e. better functional and antioxidant properties (Polyphenol 0.211 Gallic acid equivalent. $\mathrm{g}^{-1}, \mathrm{p}<0.001$ and DPPH activity $17.48 \pm 0.11 \%, \mathrm{p}<0.01$ of dried yogurt). In addition, Spirulina platensis enriched soy yogurt exhibited an amorphous type molecular structure in all four types of drying methods adopted. It could be concluded that the freeze-drying method could produce superior quality Spirulina platensis enriched dried soy yogurt powder compared to hot-air-oven and vacuum drying, while it is highly comparable to microwave drying.
\end{abstract}

Keywords: Spirulina platensis, soy yogurt, microwave-dried, vacuum-dried, freeze-dried, antioxidan

\section{INTRODUCTION}

Dairy products are highly perishable. Milk powder has increased shelf life and stored for long period without substantial loss of quality, even at ambien temperatures (Sharma et al., 2012). The dairy-based powders are mainly employed for recombination or reconstitution purposes; whereas non-dairy based powder can be exploited for their distinct functional properties for application as a food ingredient in several "value-added foods" products. Like dairy-based powder, non- dairy based powdered ingredients are stable, produced in large amount, lactose free and convenient for storage. Nowadays one of the main areas of research in food area is the development of functional foods that provide health benefits beyond their basic nutrition. Yogurt a fermented food product has a high worldwide acceptance and considered as ideal matrices to deliver beneficial nutritional ingredients. In addition, probiotic bacteria present in yogurt decrease intestinal disorder and chronic diseases. Soy bean which is the most widely grown and utilized legumes in the world has good amino acid profile, contain higher levels of essential fatty acids, soluble fiber, vitamins, minerals, phytochemicals which include isoflavones, phytic acid and saponins which have strong antioxidant properties and have capability of lowering the cholestero level. Development of new nondairy fermented food products from soybean will provide specific health benefits beyond the conventional dairy products, which have certain limitations from nutritional health aspects. Foods prepared from non-dairy based powdered ingredients like dried soy milk are usually considered to be of lower quality. Yogurts both dairy and non-dairy types are highly nutritious food products for having principally probiotic constituents and micronutrients that really help to reduce gastro intestinal problems and blood cholesterol level in human/our system and free radicals associated diseases such as cancer and osteoporosis (Savini et al., 2013). The non-dairy yogurts particularly the soy yogurts are known for such nutritional properties. In recent years, soy yogurts have been developed with expectedly enhanced nutritional superiority by incorporating functional lipids such as Gamma linolenic acid (GLA), Eicosapentaenoic acid (EPA) and Docosahexaenoic acid (DHA) and also providing specific antioxidants such as oryzanol and lignans through specific edible oils (Sengupta et al., 2013). Soy yogurts enriched with Spirulina platensis to provide higher arginine content in protein part and antioxidant such as selenium, zinc, tocopherol etc (Morsy et al., 2014). Studies showed that viability of Spirulina platensis incorporated yogurt was higher when compared to control yogurt for both Streptococcus thermophilus and Lactobacillus bulgaricus throughout the storage period (Priyanka et al., 2013). Spirulina platensis is known to have beneficial influence on the survival of the starter bacterial culture owing to its high protein, essential fatty acids such as gamma linolenic acid, vitamins and minerals (Kavimandan and Sharma, 2015). It may be pointed out that soy yogurts having probiotic and other micronutrients can be processed particularly by various drying methodologies to powder with the retention of various nutrients that in turn can find applications in various kinds of food products.

In fact, it has been stated by some researchers recently that dried yogurts in powder form have many applications such as confectionary, savory, biscuits and cakes in bakery and in soups, dips, ready meals baby foods etc. (Jaya, 2009). In recent days, there is great emphasis on adding value to powders, and therefore, an inclusive effort from non-dairy plant and powder processors, is requisite to identify the means to add more value. Consumers are willing to pay more for soy milk powders if they can perceive high functionality and quality, as well as multifunctional properties. The present investigation aims to convert Spirulina platensis enriched soy yogurts to powder form by different drying methodologies and evaluate the microstructure and composition of the powders obtained.

\section{MATERIAL AND METHODS}

All chemicals used were, purchased from MERCK, India. Soybean seeds were purchased from the local market (New Alipore Market, Kolkata West Bengal, India). Commercially available milk curd cultures were purchased from Microbial Type Culture Collection and Gene Bank, Chandigarh [Lactobacillus delbrueckii subsp. bulgaricus (MTCC 911) and Streptococcus thermophilus (MTCC 1938)]. Spirulina platensis was used as dry biomass having composition of protein $0.28 \mathrm{~g}$, energy $1.74 \mathrm{Kcal}$, fat $0.0 \mathrm{~g}$ and carbohydrate $0.16 \mathrm{~g}$ per $500 \mathrm{mg}$ (SUNOVA SPIRULINA, Surya Herbal Ltd. Noida, India). 


\section{Preparation of soy yogurt and Spirulina platensis enriched soy yogurt}

The soy milk was made according to the procedure described by Sengupta $\boldsymbol{e t}$ al., (2013). The resultant soy milk was then homogenized in a homogenizer (REM MOTORS-RQ-122) and pasteurized at $80^{\circ} \mathrm{C}$ for $15 \mathrm{~min}$. Soy milk was then cooled down to $40^{\circ} \mathrm{C}$ for the addition of Spirulina platensis. After addition of Spirulina platensis to pasteurized soy milk (1g of dry biomass of Spirulina platensis $100 \mathrm{ml}^{-1}$ of soy milk), the mixtures of soy milk and Spirulina platensis were homogenized again in a homogenizer prior to inoculation with starter culture until the Spirulina platensis was mixed properly throughout the soy milk Soy milk mixtures were aseptically inoculated with $2 \%$ of starter (Lactobacillus delbrueckii subsp. bulgaricus, Lactobacillus planterum, Lactobacillus casei and Streptococcus thermophilus, 1:1:1:1 v/v). The inoculated soy milk containing the Spirulina platensis was then poured into $100 \mathrm{ml}$ sterile transparent food grade plastic cups with lids and incubated at $37^{\circ} \mathrm{C}$ for 24 hours. Soy yogurts obtained were stored at $4^{\circ} \mathrm{C}$ in a refrigerator for further analyses. Control soy yogurt was made by following the above procedure only except incorporation of Spirulina platensis into soy milk.

\section{Drying method of soy yogurt and Spirulina platensis enriched soy yogurt}

A quantity of $200 \mathrm{~g}$ of a fresh Spirulina platensis enriched soy yogurt was dried separately by using four different drying techniques.

\section{Hot- air -oven drying}

Spirulina platensis incorporated soy yogurt was dried in hot air oven at $60^{\circ} \mathrm{C}$ for 24 hours till constant weight was obtained. The dried soy yogurt (control) and Spirulina platensis enriched soy yogurt were sealed airtight and stored at $5^{\circ} \mathrm{C}$ until analyses.

\section{Vacuum tray drying}

Spirulina platensis enriched soy yogurt was spread over a petri dish and placed on the rack of vacuum tray dryer unit (Vacuum Oven 8" dia-12" deep, Temperature up to $150^{\circ} \mathrm{C}$, Model D- 50, India). Vacuum in the dryer was set at $758 \mathrm{~mm} \mathrm{Hg}$ and the temperature kept between $45^{\circ} \mathrm{C}$ to $60^{\circ} \mathrm{C}$. The drying was continued for 5 hours until the product became free flowing. After cooling, the soy yogurt (control) and Spirulina platensis enriched soy yogurt powder were sealed airtight and stored at $5^{\circ} \mathrm{C}$ until analyses.

\section{Microwave drying}

Spirulina platensis enriched soy yogurt was spread over a polymer plate in Microwave (SAMSUNG MW83H/XTL) and heated for 10 minutes at $60^{\circ} \mathrm{C}$. The product was then collected, powdered, cooled and was sealed airtight and stored at $5^{\circ} \mathrm{C}$ until analyses.

\section{Freeze drying}

Soy yogurt (control) and Spirulina platensis enriched soy yogurt was frozen in refrigerator at $4^{\circ} \mathrm{C}$ and then freeze-dried in a freeze dryer (Freezone plus 6 , Labconco, USA) at $-40^{\circ} \mathrm{C}$ and $0.3 \mathrm{mPa}$ until constant weight (72 hours). The dried soy yogurt (control) and Spirulina platensis enriched soy yogurt powders were sealed airtight and stored at $5^{\circ} \mathrm{C}$ until analyses.

\section{Handling packaging and grinding of dried soy yogurt (control) and Spirulina platensis enriched soy yogurt}

All the samples sealed in Ziploc bags were placed inside aluminum-coated polyethylene bags. To prevent oxidation, all the packaged samples were flushed with nitrogen gas, heat sealed and stored at $35^{\circ} \mathrm{C}$ until further analyses. Dried yogurt powders obtained from different drying processes were ground using mortar and pestle. The hot-air oven dried, vacuum dried, microwave dried and freeze dried or lyophilized powder was homogenized by a 12 mesh sized sieve.

Physical property of dried soy yogurt (control) and Spirulina platensis enriched soy yogurt

\section{Water content}

The water content of dried soy yogurt (control) and Spirulina platensis enriched soy yogurt obtained by different drying methods were determined separately using the standard oven method at $70^{\circ} \mathrm{C}$ for $24 \mathrm{~h}$ (AOAC, 1998). The drying, cooling and weighing of samples was continued until the difference between two successive weighing was less than $1 \mathrm{mg}$.
Flow ability evaluation: Carr's compressibility index (C) and Hausner ratio (H)

A quantity of $50 \mathrm{~g}$ of dried soy yogurt (control) and Spirulina platensis enriched soy yogurt was filled separately into a graduated glass cylinder and repeatedly tapped on a shaker. The sample was tapped for 500 times and repeated at 3 turns. The volume of powder after tapping and Carr's index percent was measured as equation 1. Hausner ratio is related to inter-particle friction. In this test, values less than 1.25 indicate good flow $(\approx 20 \%$ Carr $)$, a value greater than 1.5 indicate poor flow $(\approx 33 \%$ Carr $)$.

$$
\text { Carr's compressibility index }=\frac{\text { Bulked density }}{\text { Tapped density }}
$$

Hausner ratio $=\frac{\text { Tapped density }}{\text { Bulked density }}$

Antioxidant property of dried soy yogurt (control) and Spirulina platensis enriched soy yogurt

\section{Polyphenol assay}

Estimation of polyphenols was determined using Folin-Ciocalteu reagent (Singleton and Rossi, 1965) with some modification. $0.1 \mathrm{ml}$ dried soy yogurt (control) and Spirulina platensis enriched soy yogurt $\left(10 \mathrm{mg} \mathrm{ml}^{-1}\right.$ in distilled water) was extracted separately for $2 \mathrm{~h}$ at room temperature on a mechanical shaker. To them, $1 \mathrm{ml}$ of Folin-Ciocalteu reagent (1:2 dilution) and $2 \mathrm{ml}$ of $10 \%$ $\mathrm{Na}_{2} \mathrm{CO}_{3}$ was added. The mixture was centrifuged at $20,000 \times \mathrm{g}$ for $20 \mathrm{~min}$, and the supernatant was decanted and filtered through Whatman No.1 filter paper. The absorbance of the clear supernatant solution was measured at $765 \mathrm{~nm}$ (V-630 UV-VIS Spectrophotometer, JASCO). Gallic acid was used as a standard. Each sample was analyzed twice with duplicates. Results were expressed as mg GAE $100 \mathrm{~g}^{-1}$ dry weight.

\section{1, 1-diphenyl-2-picrylhydrazyl (DPPH) assay}

DPPH radical scavenging activity was determined using 1, 1-diphenyl-2 picrylhydrazyl (DPPH) assay (Bansal et al., 2014) with some modification. 0.5 $\mathrm{ml}$ dried soy yogurt (control) and Spirulina platensis enriched soy yogurt (10 mg $\mathrm{ml}^{-1}$ in distilled water) was added separately to $2.5 \mathrm{ml} \mathrm{DPPH}$ reagent $(0.2 \mathrm{mM})$. The reaction mixture was kept in dark at ambient temperature for $30 \mathrm{~min}$. The absorbance was measured using a spectrophotometer at $517 \mathrm{~nm}$ (V-630 UV-VIS Spectrophotometer, JASCO). DPPH radical scavenging activity (\%) was calculated using following formula:

$$
\% \text { Inhibition }=\frac{(\text { Absorbance } 517 \text { control }- \text { Absorbance } 517 \text { extract })}{(\text { Absorbance } 517 \text { control })} \times 100
$$

\section{Ferric reducing antioxidant power (FRAP) assay}

Reducing power was determined using ferric reducing antioxidant power (FRAP assay (Barahona et al., 2011) with some modification. $0.5 \mathrm{ml}$ dried soy yogurt (control) and Spirulina platensis enriched soy yogurt $\left(10 \mathrm{mg} \mathrm{ml}^{-1}\right.$ in distilled water) was added separately to $2.5 \mathrm{ml}$ FRAP reagent The FRAP reagent consist of $300 \mathrm{mM}$ acetate buffer $(3.1 \mathrm{~g}$ sodium acetate $+16 \mathrm{ml}$ glacial acetic acid, made up to $1 \mathrm{~L}$ with distilled water; $\mathrm{pH}=3.6), 10 \mathrm{mM}$ TPTZ in $40 \mathrm{mM} \mathrm{HCl}$ and 20 $\mathrm{mM} \mathrm{FeCl} 3 \cdot 6 \mathrm{H}_{2} \mathrm{O}$ in the ratio of $10: 1: 1$. The reaction mixture was kept in dark at ambient temperature for $10 \mathrm{~min}$. The absorbance was measured using a spectrophotometer at $593 \mathrm{~nm}$ (V-630 UV-VIS Spectrophotometer, JASCO) Reducing power was calculated using following formula:

$$
\text { Reducing power }=\text { Abs593 sample }- \text { Abs } 593 \text { FRAP reagent }
$$

\section{Carotenoid estimation}

$0.5 \mathrm{ml}$ dried soy yogurt (control) and Spirulina platensis enriched soy yogurt (10 $\mathrm{mg} \mathrm{ml}^{-1}$ in distilled water) was centrifuged separately at $3000 \mathrm{rpm}$ for 5 minutes The pellet was washed with distilled water 2-3 times. To the pellet, 2-3 ml of acetone $(85 \%)$ was added, which was then subjected to repeated freezing and thawing. The suspension was centrifuged and the supernatant containing pigment was collected. The extraction was repeated till the supernatant became colorless, for complete recovery of carotenoid. The pooled fractions of supernatants were made-up to a final known volume. The absorbance was taken at $450 \mathrm{~nm}$ using $85 \%$ acetone as blank and the total amount of carotenoids was calculated in $\mathrm{mg} \mathrm{g}$ ${ }^{1}$ as follows (Saleh et al., 2011).

$$
C=\frac{D \times V F}{2500 \times 100}
$$


$\mathrm{D}=\mathrm{OD}$ at $450 \mathrm{~nm}, \mathrm{~V}=$ Volume of the extract, $\mathrm{F}=$ Dilution factor (Assuming that average extinction coefficient is 2500 )

Color parameter of dried soy yogurt (control) and Spirulina platensis enriched soy yogurt

Color parameters were determined using colorimeter (Minolta Chroma meter CR-300, USA). Dried soy yogurt (control) and Spirulina platensis enriched soy yogurt were poured into a clear glass petri dish and color coordinate values (lightness, $\mathrm{L}^{*}$, redness, $\mathrm{a}^{*}$, and yellowness, $\mathrm{b}^{*}$ ) were recorded separately.

X-Ray Diffraction pattern of dried soy yogurt (control) and Spirulina platensis enriched soy yogurt

X-ray diffraction patterns of dried soy yogurt (control) and Spirulina platensis enriched soy yogurt were obtained at room temperature using a Rigaku Multiflex powder diffractometer ( $\mathrm{CuK} \alpha$ radiation generator) operated at a voltage of $40 \mathrm{kV}$. Powdered, dried yogurt samples were analyzed separately at two theta $(2 \theta)$ angle, range of $10-30$

Morphology analysis of dried soy yogurt (control) and spirulina platensi enriched soy yogurt using scanning electron microscopic analysis (SEM)

Dried soy yogurt (control) and Spirulina platensis enriched soy yogurt at micro level were examined separately by scanning electron microscope for desired structural properties. The dried Soy yogurt and Spirulina platensis incorporated soy yogurt was previously fixed on an iron stub and then made electrically conductive by coating in a vacuum chamber with a thin layer of gold for $40 \mathrm{~s}$ The pictures were taken at an excitation voltage of $15 \mathrm{kv}$ at different magnifications varying from $1500-1600$

\section{Statistical analysis}

Statistical analysis was performed by using analysis of variance (ANOVA) and the means were compared across groups by Tukey test. All analyses were carried out in triplicates repeated at least twice with the Origin Pro 8 and the significant differences were determined at $\mathrm{p} \leq 0.05$

\section{RESULTS AND DISCUSSION}

Soy yogurt is generally dried by freeze, spray, microwave and vacuum drying Fazaeli et al., (2012) observed that among the different drying methods freezedrying is one of the most advanced methods for drying food products since it retains taste, aroma, flavor, color and the nutritional quality. In addition (Rybka and Kailasapathy, 1997) also observed that freeze-dried soy yogurt is the authentic product in comparison to yogurt obtained using other conventional drying methods. Another study revealed that there is no significant change on the final contents of total protein casein, serum and non-protein nitrogen obtained from freeze-drying at $-40^{\circ} \mathrm{C}$. Radaeva et al., (1975) found that survival of lactic acid bacteria in yogurt was $50-60 \%$ during freeze-drying at $-40^{\circ} \mathrm{C}$. Kitawaki $\boldsymbol{e}$ al., (2009) showed that freeze-dried soy yogurt is beneficial in preventing hepatic lipid accumulation in rats. From the above literature, it was revealed that that freeze drying best choice of the drying methods tested for evaluation of the soy yogurt. Other drying techniques also work well but should not be used for soy yogurts due to sensitivity to oxidation.

Physical property of dried soy yogurt (control) and Spirulina platensi enriched soy yogurt

The physical properties of yogurt powder are shown in Tab 1. No significant differences were observed in water content between Product-I and Product-V. Data showed that vacuum dried yogurt sample had the significantly highes $(3.67 \pm 0.67 \%, \mathrm{p}<0.01)$ and the microwave dried had the significantly lowest moisture content $(1.89 \pm 0.55 \%, \mathrm{p}<0.001)$ in comparison with Product-I. Hot ai and freeze dried yogurt samples had moisture close to each other. During vacuum drying, the yogurt sample was expanded under the vacuum.

Table 1 Selected physical properties of dried soy yogurt (control) and Spirulina platensis enriched soy yogurt

\begin{tabular}{|c|c|c|c|c|c|}
\hline Materials & Water content $(\%)$ & Bulk density $\left(\mathrm{g} \mathrm{cm}^{-3}\right)$ & Tapped density $\left(\mathrm{g} \mathrm{cm}^{-3}\right)$ & $\mathbf{C}$ & HR \\
\hline Product-I (control) & $2.55 \pm 0.61$ & $0.22 \pm 0.08$ & $0.41 \pm 0.49$ & $53.65 \pm 0.21$ & $1.86 \pm 0.48$ \\
\hline Product-II & $2.62 \pm 0.56$ & $0.39 \pm 0.04^{\mathrm{a}}$ & $0.59 \pm 0.04^{\mathrm{a}}$ & $66.10 \pm 0.05^{\mathrm{a}}$ & $1.51 \pm 0.32^{\mathrm{a}}$ \\
\hline Product-III & $3.67 \pm 0.67^{\mathrm{b}}$ & $0.32 \pm 0.04^{\mathrm{a}}$ & $0.58 \pm 0.03^{\mathrm{b}}$ & $55.17 \pm 0.06^{\mathrm{a}}$ & $1.81 \pm 0.11^{\mathrm{b}}$ \\
\hline Product-IV & $1.89 \pm 0.55^{\mathrm{c}}$ & $0.29 \pm 0.07$ & $0.44 \pm 0.09^{\mathrm{b}}$ & $65.90 \pm 0.03^{\mathrm{a}}$ & $1.51 \pm 0.23^{\mathrm{b}}$ \\
\hline Product-V & $2.52 \pm 0.98$ & $0.38 \pm 0.02^{\mathrm{a}}$ & $0.47 \pm 0.11^{\mathrm{a}}$ & $66.66 \pm 0.03^{\mathrm{a}}$ & $1.23 \pm 0.16^{\mathrm{c}}$ \\
\hline
\end{tabular}

The data are mean \pm S.D. and significantly different at ${ }^{\mathrm{a}} P<0.05,{ }^{\mathrm{b}} \mathrm{P}<0.01$ and ${ }^{\mathrm{c}} \mathrm{P}<0.001$ vs. Product-I, SP- Spirulina platensis, C- Carr's
Index, HR- Hausner ratio, SP- Spirulina platensis; Product-I: soy yogurt (freeze dried), Product-II: SP soy yogurt (hot air oven dried),Product-III: SP soy yogurt (vacuum dried), Product-IV: SP soy yogurt (microwave dried), Product-V: SP soy yogurt freeze dried.

Compressibility is the ability to reduce volume by tapping, developed by Carr (1965) as an average and indirect measure of cohesion forces. The flowability scale is "universal", as it has been constructed on previous measurements of 300 different powders, from [0-19] very poor, [20-39] poor, [40-59] not good, [6069] normal, [70-79] good, [80-89] fairly good and [90-100] very good. All the yogurt powders that we had dried had been under the value of 60 , except Product-I and Product-III corresponding to "normal flowability". Compressibility for the product was followed the same tendency as the Hausner ratio, declining when vacuum dried applied. Note that a Product-II, IV and V presented a norma flow, without special problems with a flowability index value of above 60 . Only the Product-III presented significantly worse flowability $(55.17 \pm 0.06, \mathrm{p}<0.05)$ than Product-I. Product-I could not present good flowability because of its low content of carbohydrate and protein percentage. Product-IV presented extreme values for low Hausner ratio $(1.23 \pm 0.16, \mathrm{p}<0.001)$, together with the best flowability index $(66.6 \pm 0.03 \mathrm{p}<0.05)$. This product should therefore present high internal homogeneity due to Spirulina platensis incorporation and quite "exceptional properties" within the drying techniques studied. This flowability index value is quite unusual for non-dairy powder.

Antioxidant property of dried soy yogurt (control) and Spirulina platensis enriched soy yogurt

Antioxidant property of dried soy yogurt (control) and Spirulina platensi enriched soy yogurt affected by different drying methods is illustrated in Fig 1.
Spirulina, are believed to be a rich source of polyphenol antioxidants. Spirulina platensis fortification was found to bring about a significant increase in the antioxidant capacity, but drying was found to bring about a reduction. Total poly phenolic content was significantly the highest in product-IV $\left(0.185 \mathrm{GAE} \mathrm{g}^{-1}\right.$, $\mathrm{p}<0.05$ and Product-V $\left(0.211 \mathrm{GAE} \mathrm{g}^{-1}, \mathrm{p}<0.05\right.$ of dried yogurt $)$. Product- $\mathrm{V}$ showed significantly highest total phenolic content $\left(0.211 \mathrm{GAE} \mathrm{g}^{-1}, \mathrm{p}<0.001\right.$ of dried yogurt), in comparison with Product-II and Product-III. However, not a big difference in percentage increase of activity was observed for all four drying treatment, with Spirulina platensis fortification. In the FRAP assay, the antioxidant activity of Product-II and Product-III was not affected by respective drying treatments. On the contrary, in the DPPH assay, the antioxidant activity was significantly influenced for Product-II $(14.58 \pm 0.04 \%, \mathrm{p}<0.05$ of dried yogurt) and Product-III (14.26 $\pm 0.02 \%, \mathrm{p}<0.05$ of dried yogurt). DPPH radical scavenging activities were not significantly different between Product-IV $(15.67 \pm 0.01 \%, \mathrm{p}<0.01$ of dried yogurt) and Product-V $(17.48 \pm 0.11 \%, \mathrm{p}<0.01$ of dried yogurt), while both were significantly higher than Product-II and ProductIII. The results showed (Fig 1) that the total carotenoid content of powders obtained by Spirulina platensis incorporated soy yogurt was significantly influenced by different drying method The highest carotenoid content $(5.77 \mathrm{mg} \mathrm{g}$ ${ }^{1}$ of dried yogurt) was for Product-III whilst the Product-II that was hot air dried had the lowest carotenoid content $\left(3.27 \mathrm{mg} \mathrm{g}^{-1}\right.$ of dried yogurt). 

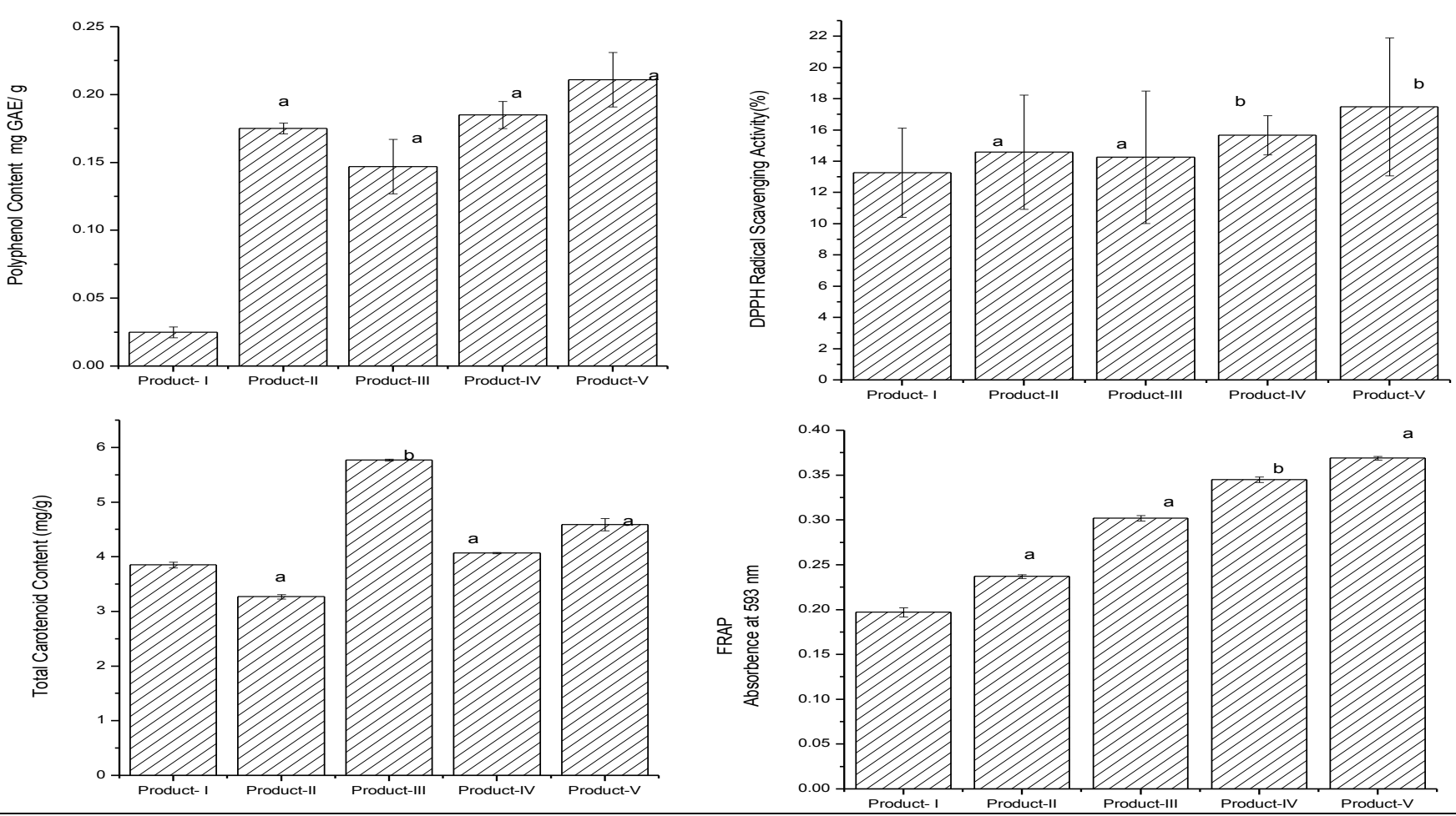

Fig 1 Polyphenol content, DPPH radical scavenging activity, FRAP and carotenoid content of dried soy yogurt (control) and Spirulina platensis enriched soy yogurt.SP- Spirulina platensis, C- Carr's Index, HR- Hausner ratio, SP- Spirulina platensis; Product-I: soy yogurt (freeze dried), Product-I: soy yogurt (freeze dried), Product-II: SP soy yogurt (hot air oven dried),Product-III: SP soy yogurt (vacuum dried), Product-IV: SP soy yogurt (microwave dried), ProductV: SP soy yogurt freeze dried. GAE: Gallic acid equivalent, DPPH: 1, 1-diphenyl-2-picrylhydrazyl, FRAP: Ferric reducing antioxidant power.

There was no significant difference between Product-IV and Product-V in terms of total carotenoid content. It is evident that vacuum drying was more effective in the retention of total carotenoid than air-drying. Therefore, it can be concluded that the main reason for carotenoid degradation is due to loss of anthocyanin. It is clear that the freeze-drying process can substantially preserve the nutritional value of Spirulina platensis in terms of total antioxidant activity. From the results of the various tests in the study, the quality of freeze-dried Spirulina platensis incorporated soy yogurt powder products is seen to be highest, followed by microwave-dried powder, vacuum-dried powder, air-dried powder, and then powders from soy yogurt as control.

Color parameter of dried soy yogurt (control) and Spirulina platensis enriched soy yogurt

It was observed from Tab 2 that the Lightness $\left(\mathrm{L}^{*}\right)$, redness $\left(\mathrm{a}^{*}\right)$ yellowness $\left(\mathrm{b}^{*}\right)$ and hue angle $(\mathrm{h})$ were significantly different among the dried Spirulina platensi enriched soy yogurt. The highest $\mathrm{L}^{*}$ value $(59.34 \pm 2.65, \mathrm{p}<0.01)$ and lowest $\mathrm{a}^{*}$ value $(-17.48 \pm 0.98, \mathrm{p}<0.01)$ was observed in Product-V in comparison with Product-II, III and IV. The highest L* value could attribute to minimal color deterioration. Freeze-drying can retarded oxidation and other chemical reactions, and thus minimal color deterioration (Ratti et al., 2001). Meanwhile, the lowest $\mathrm{L}^{*}$ value $(40.57 \pm 4.67, \mathrm{p}<0.05)$ and highest $\mathrm{a}^{*}$ value $(-14.58 \pm 5.58, \mathrm{p}<0.05)$ was observed in Product-II. The lowest $\mathrm{L}^{*}$ value could attribute to color degradation. Oven drying can cause oxidative degradation, and thus lead to color change (turn into darker color). No significant difference was observed in $b^{*}$ value (yellowness) between Product-IV and Product-V while there was a highly significant difference between Product-II and Product-III (Tab 2). Product-IV obtained a higher $\mathrm{h}$ value $(150.77 \pm 7.64, \mathrm{p}<0.05)$ compared to Product-II, II and $\mathrm{V}$ suggesting that microwave dried Product-IV is more vivid in its dark green color implying that it will be more attractive and appealing to consumers. The overall distinct vivid dark green color of the Product-IV may be indicative of high chlorophyll retention. The minimal color change of product produced by microwave dried Product-IV and freeze-dried Product-V suggests the appropriateness of these processes to produce high quality products.

Table 2 Color measurements of soy yogurt and Spirulina platensis incorporated soy yogurt powder obtained by different drying methods

\begin{tabular}{lllll}
\hline Materials & L $^{*}$ & $\mathbf{a}^{*}$ & $\mathbf{b}^{*}$ & \multicolumn{1}{c}{ h } \\
\hline Product- I (control) & $75.64 \pm 6.27$ & $0.26 \pm 2.85$ & $12.25 \pm 0.87$ & $83.85 \pm 9.25$ \\
Product-II & $40.57 \pm 4.67^{\mathrm{a}}$ & $-14.58 \pm 5.58^{\mathrm{a}}$ & $6.81 \pm 0.54^{\mathrm{a}}$ & $130.11 \pm 8.31^{\mathrm{c}}$ \\
Product-III & $51.74 \pm 6.25^{\mathrm{c}}$ & $-14.26 \pm 3.54^{\mathrm{a}}$ & $6.35 \pm 0.34^{\mathrm{c}}$ & $143.79 \pm 8.46^{\mathrm{b}}$ \\
Product-IV & $46.27 \pm 3.84^{\mathrm{a}}$ & $-15.67 \pm 1.26^{\mathrm{c}}$ & $7.65 \pm 0.87^{\mathrm{b}}$ & $150.77 \pm 7.64^{\mathrm{a}}$ \\
Product-V & $59.34 \pm 2.65^{\mathrm{b}}$ & $-17.48 \pm 0.98^{\mathrm{b}}$ & $7.29 \pm 0.69^{\mathrm{b}}$ & $144.07 \pm 6.47^{\mathrm{b}}$ \\
\hline
\end{tabular}

The data are mean \pm S.D. and significantly different at ${ }^{\mathrm{a}} \mathrm{P}<0.05,{ }^{\mathrm{b}} \mathrm{P}<0.01$ and ${ }^{\mathrm{c}} \mathrm{P}<0.001$ vs. Product-I, SP- Spirulina platensis, C- Carr's Index, HR- Hausner ratio, SP- Spirulina platensis; Product-I: soy yogurt (freeze dried), Product-II: SP soy yogurt (hot air oven dried),Product-III: SP soy yogurt (vacuum dried), Product-IV: SP soy yogurt (microwave dried), Product-V: SP soy yogurt freeze dried.

X-ray diffraction patterns of dried soy yogurt (control) and Spirulina platensis enriched soy yogurt

The X-ray diffraction patterns of the soy yogurt (control) and Spirulina platensis enriched soy yogurt gave valuable information about structural aspects of the Spirulina platensis (Fig 2). In this study, Product-II, III, IV and V had a significant influence on the degree of dispersion and aggregation in soy yogurt. $\mathrm{XRD}$ is a very common technique used to confirm the crystalline-amorphous state of dried yogurt powder. Dried yogurt obtained from all the form of drying methods exhibited amorphous nature. Amorphous products produce a broad background pattern while crystalline material exhibits sharp peaks while. Crystalline nature obtained from XRD is expected due to presence of raffinose and stachyose sugar. Rapid drying of low molecular weight sugar present in yogurt tends to produce amorphous metastable state of dried products due to insufficient time to crystallize. Drying methods of yogurt showed no crystalline peaks formation. In case of freeze drying the temperature of yogurt was less than $50^{\circ} \mathrm{C}$. Crystal formation generally occurs above $50^{\circ} \mathrm{C}$. Thus in case of freezedrying, less crystalline is preferred. These figures exhibit essentially similar diffraction patterns ( $2 \theta$ values) for all samples suggesting that dried yogurts did not undergo any structural modifications (Fig 2). However, a major reduction in relative intensities of their peaks particularly for Product-II and Product-IV might be due to reduction in crystallinity and presence of amorphous state in the samples. Therefore, it was expected that in the case of drying by freeze drier, Spirulina platensis incorporated dried soy yogurt particles with less crystallinity were produced. However, it was interesting to note that the intensity count for Product-II as shown in the diffractograms was significantly lower compared to the other three powder products. 


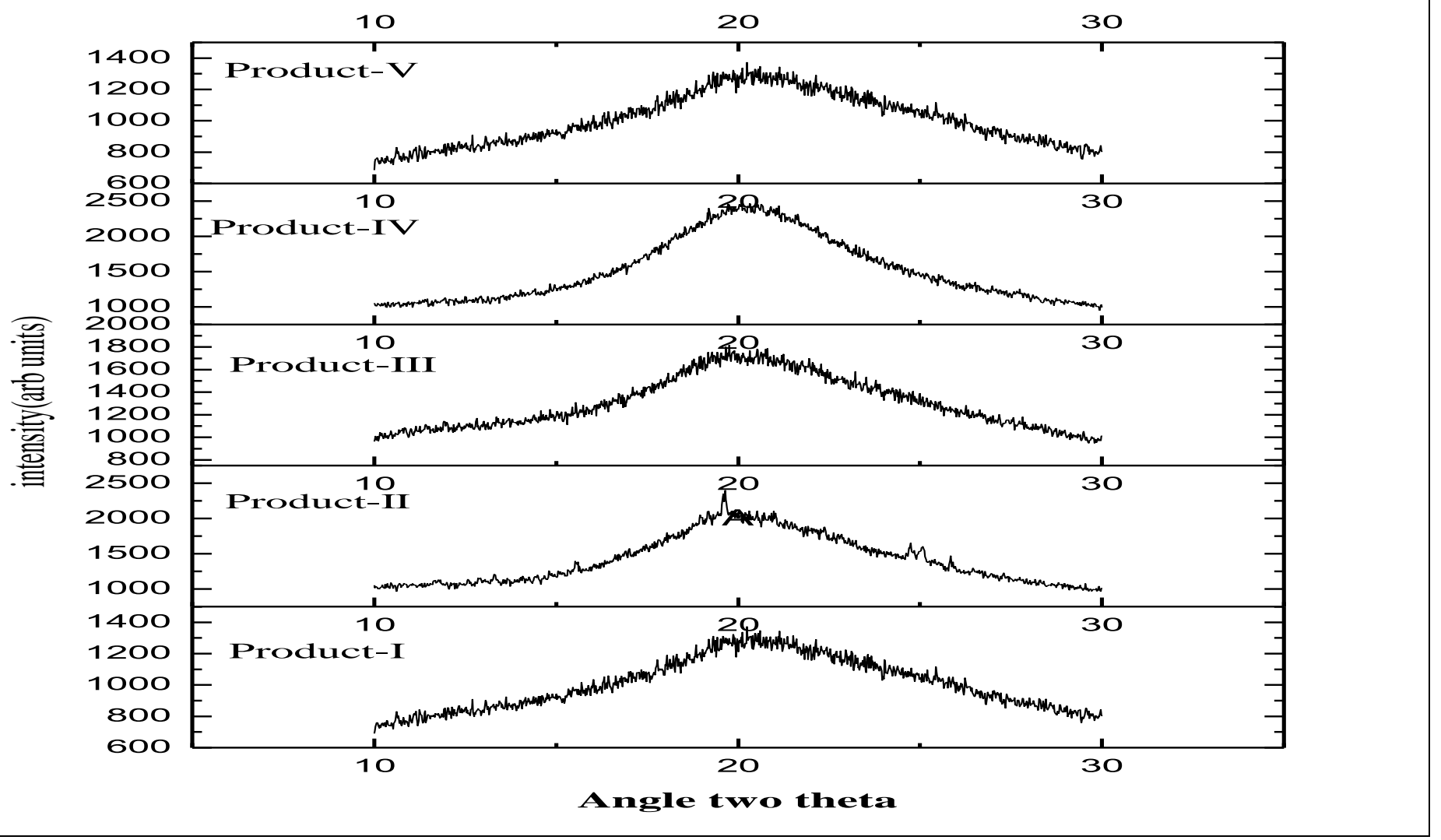

Fig 2 X-ray diffraction patterns of dried soy yogurt (control) and Spirulina platensis enriched soy yogurt

SP- Spirulina platensis, C- Carr's Index, HR- Hausner ratio, SP- Spirulina platensis; Product-I: soy yogurt (freeze dried), Product-II: SP soy yogurt (hot air oven dried), Product-III: SP soy yogurt (vacuum dried), Product-IV: SP soy yogurt (microwave dried), Product-V: SP soy yogurt freeze dried.

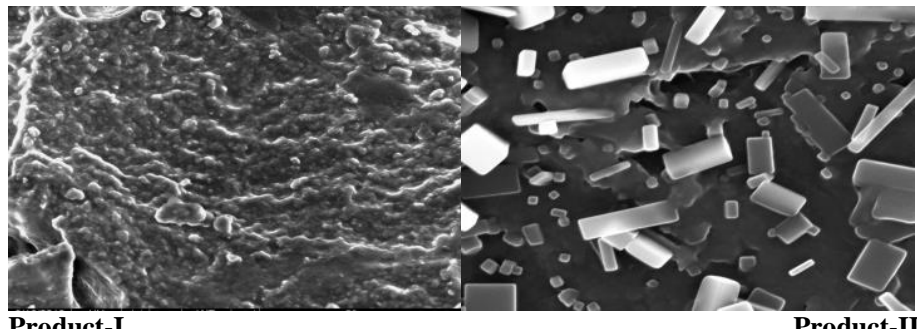

Product-I

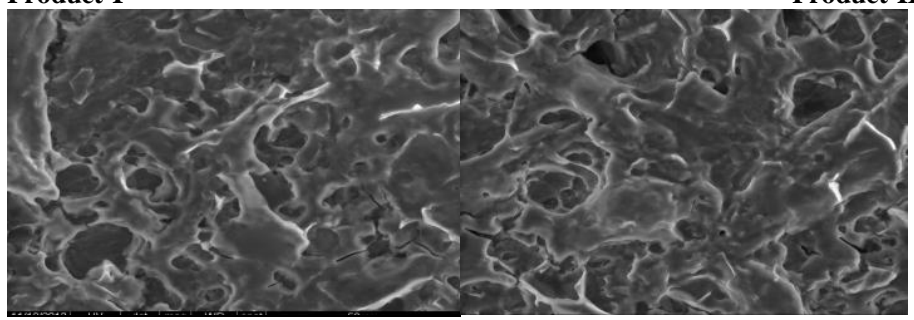

\section{Product-III}

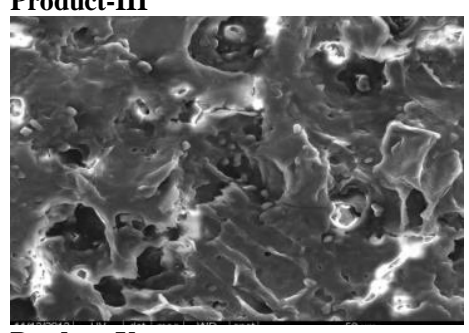

Product-V

Fig 3 Scanning electron micrographs (SEM) of soy yogurt and Spirulina platensis enriched soy yogurt (magnification of $800 \times 20 \mathrm{kV}$ ).

SP- Spirulina platensis; Product-I: soy yogurt (freeze dried), Product-II: SP soy yogurt (hot air oven dried),Product-III: SP soy yogurt (vacuum dried), ProductIV: SP soy yogurt (microwave dried), Product-V: SP soy yogurt freeze dried.Product- I: soy yogurt-freeze dried; Product-II: SP soy yogurt hot air oven
Dried; Product-III: SP soy yogurt vacuum dried; Product-IV: SP soy yogurt microwave dried; Product-V: SP soy yogurt freeze dried.

Scanning electron micrographs (SEM) of dried soy yogurt (control) and Spirulina platensis enriched soy yogurt

Scanning electron micrographic studies of soy yogurt and Spirulina platensis enriched soy yogurt are shown in Fig 3. The microstructure of Product-II was compact and exhibited irregular particles with sharp edges and considerable indentation because of crushing into powder. The microstructure of Product-III and Product-IV was smooth, and flaky with uniform thickness. Product-V showed a skeletal like structure and was more porous than the other Product. This is due to the ice in the material during freeze drying helps prevent shrinkage and collapse of the structure and shape resulting in an insignificant change in volume.

\section{CONCLUSION}

The primary objective of manufacturing dried yogurt in powder form is to increase the shelf life of yogurt. Freeze-drying is a physical phenomenon of sublimation, which consists of first freezing the yogurt and then reducing the surrounding presence to allow the frozen yogurt to sublimate directly for solid phase to gaseous phase without passing through the liquid state. Freeze dried products have very good capacity to take up water again since have extremely large internal surface area and they. Thus, freeze-dried products can be stored from very long time without too much loss of nutritional quality. Thus in freeze drying, products ingredients are preserved with good nutritional value. Moreover, freely dried yogurt retained flavor, color, taste in comparison to yogurt obtained using other drying methods (Fazaeli et al., 2012) and also has better rehydration property. Absence of air causes very less deterioration of food product. Another study revealed that freeze-drying did not affect the final content of protein casein, serum and non-protein nitrogen. Again, it was observed that in yogurt 50- 60\% of lactic acid bacteria were survived during freeze-drying (Radaeva, 1975). That is why freeze-drying method is best drying methods. Drying techniques were shown to exert significant effect on Spirulina platensis enriched dried soy yogurt. Freeze-drying was most suitable method to produce high quality dried Spirulina platensis enriched soy yogurt, i.e. better functional and antioxidant properties. Overall, our study concludes that the freeze drying method can produce superior quality Spirulina platensis enriched dried soy yogurt powder compared to hot-airoven and vacuum drying, while it is highly comparable to microwave drying. The study provides an opportunity to the powder processing industry in selecting a 
better drying technique that can be utilized for the production of high quality yogurt powder, a complementary formulated as a tablet from soy yogurt powder is possible and also may be therapeutically effective against lactose-intolerance syndrome and preventing antibiotic-associated diarrhea.

\section{REFERENCES}

AOAC, 1998. Official Methods of Analysis. Association of Official Analytical Chemists, Washington, DC, USA.

Bansal, V., Sharma, H. K., \& Nanda, V. (2014). Optimisation of spray drying process parameters for low-fat honey-based milk powder with antioxidant

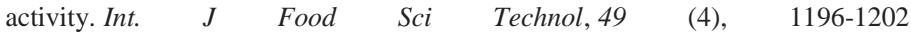
http://dx.doi.org/10.1111/ijfs.12416

Barahona, T., Chandía, N.P., Encinas, M.V., Matsuhiro, B. \& Zúñiga, E.A. (2011). Antioxidant capacity of sulfated polysaccharides from seaweeds. A $\begin{array}{llll}\text { kinetic approach. Food Hydrocoll, } & 25, & 529-535\end{array}$ http://dx.doi.org/10.1016/j.foodhyd.2010.08.004

Carr, R.L. (1965). Evaluating flow properties of solids. Chem. Eng. J. 72, 163 168

Fazaeli, M., Tahmasebi, M. \& Emam.Djomeh, Z. (2012). Characterization of food texture: application of Microscopic technology. Current Microscopy Contributions to Advances in Science and Technology,1, 855-871.

Kavimandan, A., \& Sharma S. (2015). Role of Spirulina platensis as an enhancer of probiotic organisms in whey. International journal of dairy science research, 3(1), 1-6

Kitawaki, R., Nishimura, Y.Y., Takagi, N., Iwasaki, M., Tsuzuki, K., \& Fukuda, M. (2009). Effects of Lactobacillus Fermented Soymilk and Soy Yogurt on Hepatic Lipid Accumulation in Rats Fed a Cholesterol-Free Diet. Biosci. Biotechnol. Biochem., 73 (7), 1484-1488. http://dx.doi.org/10.1271/bbb.80753

Malik, P., Kempanna, C., Narasimha, M., \& Anjum. (2013). Quality Characteristics of Yoghurt Enriched with Spirulina Powder. Mysore J. Agric, Sci., 47 (2), 354-359.

Morsy, O.M., Sharoba, A.M., EL-Desouky, A.I., Bahlol, H.E.M. \& Abd E

Mawla, E.M. Production and evaluation of some extruded food products using spirulina algae. Annals of Agric. Sci., 52(4,) 2014.

Jaya. S. (2009) Microstructure Analysis of Dried Yogurt: Effect of Different Drying Methods. Int J Food Prop, 12, 469-481. http://dx.doi.org/10.1080/10942910701772071

Ratti, C. (2001). Hot air and freeze-drying of high-value foods - a review. $J$. Food Eng, 49, 311-319. http://dx.doi.org/10.1016/S0260-8774(00)00228-4

Saleh, A.M., Dhar, D.W., and Singh, P.K. (2011). Comparative pigment profiles of different Spirulina strains. R. in Biotech, 2(2); 67-74.

Savini, I., Catani, M. V., Evangelista, D., Gasperi, V., \& Avigliano, L. (2013) Obesity-Associated Oxidative Stress: Strategies Finalized to Improve Redox
State. Int
J Mol
Sci, 14(5),
$10497-10538$

http://dx.doi.org/10.3390/ijms140510497

Sengupta, S., Bhowal, J., Bhattacharyya, D.K. (2013). Development of new kinds of Soy yogurt containing functional lipids as superior quality food. Annals of Biological Research, 4,144-151.

Sharma, A., Jana, A.H., \& Chavan , R. S.(2012). Functionality of Milk Powders and Milk-Based Powders for End Use Applications-A Review. Comp Rev Food Sci Food, 11, 518-528. http://dx.doi.org/10.1111/j.1541-4337.2012.00199.x

Radaeva, I.A., Shul'kina, S.P., Kocherga, S.I. \& Efron, B.G. (1975). Effect of freezing regimes in freeze-drying on yogurt quality. Molochnaya Promyshlennost, 5, 22-23.

Rybka, S. \& Kailasapathy, K.(1997). Effect of freeze drying and storage on the microbiological and physical properties of AB yogurt". Milchwissenschaft, 52 390-394.

Singleton, V.L. \& Rossi, J. A. (1965). Colorimetry of total phenolics with phosphomolybdic-phosphotungtic acid reagent. Am. J. Enol. Vitic, 144-158 16, 144-158. 